\title{
ANALYTIC INTEGRABILITY OF QUADRATIC-LINEAR POLYNOMIAL DIFFERENTIAL SYSTEMS
}

\author{
JAUME LLIBRE ${ }^{1}$ AND CLÀUDIA VALLS ${ }^{2}$
}

\begin{abstract}
For the quadratic-linear polynomial differential systems with a finite singular point, we classify the ones which have a global analytic first integral, and provide the explicit expression of their first integrals.
\end{abstract}

\section{INTRODUCTION}

For a two-dimensional system the existence of a first integral determines completely its phase portrait. For such systems the notion of integrability is based on the existence of a first integral. Then a natural question arises: Given a system of ordinary differential equations in $\mathbb{R}^{2}$ depending on parameters, how to recognize the values of such parameters for which the system has a first integral?

The planar integrable systems which are not Hamiltonian, i.e. the systems in $\mathbb{R}^{2}$ that cannot be written as $x^{\prime}=-\partial H / \partial y, y^{\prime}=\partial H / \partial x$ for some function $H: \mathbb{R}^{2} \rightarrow \mathbb{R}$ of class $C^{2}$, are in general very difficult to detect.

Let $P$ and $Q$ be two real polynomials in the variables $x$ and $y$, then we say that the system

$$
x^{\prime}=P(x, y), \quad y^{\prime}=Q(x, y),
$$

is a quadratic polynomial differential system if the maximum of the degrees of the polynomials $P$ and $Q$ is two.

Quadratic polynomial differential systems have been investigated intensively, and more than one thousand papers have been published about these systems (see for instance $[3,17,18,19])$, but the problem of classifying all the integrable quadratic polynomial differential systems remains open. For more information on integrable differential systems in dimension 2, see for instance [5].

Let $\mathbb{R}[x, y]$ be the ring of polynomials in the variables $x$ and $y$ with coefficients in $\mathbb{R}$. In this paper we deal with quadratic-linear polynomial differential systems in $\mathbb{R}^{2}$, i.e. systems of the form

$$
\frac{d x}{d t}=x^{\prime}=P(x, y) \quad \frac{d y}{d t}=y^{\prime}=Q(x, y),
$$

where $P, Q \in \mathbb{R}[x, y]$ with $\operatorname{deg} P=2$ and $\operatorname{deg} Q=1$. In what follows we will denote them simply by quadratic-linear systems.

Here a global analytic first integral or simply an analytic first integral is a nonconstant analytic function $H: \mathbb{R}^{2} \rightarrow \mathbb{R}$, whose domain of definition is the whole $\mathbb{R}^{2}$

1991 Mathematics Subject Classification. Primary 34C05, 34A34, 34C14.

Key words and phrases. analytic integrability, quadratic systems, quadratic vector fields, quadratic-linear systems, quadratic-linear vector fields. 
and it is constant on the solutions of system (1). This last assertion means that for any solution $(x(t), y(t))$ of $(1)$ we have

$$
\frac{d H}{d t}(x(t), y(t))=\frac{\partial H}{\partial x} x^{\prime}+\frac{\partial H}{\partial y} y^{\prime}=0 .
$$

We note that a complete characterization of the global analytic first integrals of polynomial differential systems has been made for very few families of differential systems (see, for example [13]) where the authors provide the complete characterization of all Lotka-Volterra systems in $\mathbb{R}^{2}$ with 6 parameters having a global analytic first integral. In [4] the authors classify the quadratic polynomial differential systems having a polynomial first integral.

The goal of this paper is to classify all the quadratic-linear polynomial differential systems having a finite singular point and a global analytic first integral. Additionally we provide an explicit normal form of these systems and of their analytic first integrals. This is an important subclass of all the quadratic systems, and so is a good first step towards the final goal of classifying all quadratic polynomial systems having a global analytic first integral but this final objective looks now very difficult.

By Proposition 3 we only need to consider quadratic-linear polynomial differential systems of the form

$$
x^{\prime}=P(x, y)=b x+c y+d x^{2}+e x y+f y^{2}, \quad y^{\prime}=Q(x, y),
$$

where $P(x, y) \neq b x+c y$, and $Q(x, y)$ is either $x$, or $y$. Moreover

(S1) if $Q(x, y)=y$, then $P(x, y) \neq c y+e x y+f y^{2}$; and

(S2) if $Q(x, y)=x$, then $P(x, y) \neq b x+d x^{2}+e x y$.

Note that we have avoided: first that the differential system be linear, and second the situations in which the polynomials $P(x, y)$ and $Q(x, y)$ have a common factor, because then with a rescaling of the time variable we can reduce the problem to a non-interesting linear differential system.

We also do not consider in (3) the case $Q(x, y)=0$ because it clearly has the global analytic first integral $y$.

Through the paper $\mathbb{Z}^{+}$will denote the set of non-negative integers, $\mathbb{Z}^{-}$will denote the set of negative integers, $\mathbb{Q}^{+}$will denote the set of non-negative rationals and $\mathbb{Q}^{-}$will denote the set of negative rationals.

The main results of this paper are the following.

Theorem 1. The quadratic-linear polynomial differential systems(3) satisfying (S1) and having a global analytic first integral $H$ are the following:

(1) $b=d=0$ having $H=\exp (-e y)\left(e^{2} x+e f y+c e+f\right)$.

(2) $d=0, b=-p / q \in \mathbb{Q}^{-}$and $e=0$ having $H=y^{p}\left(\frac{c q y}{p+q}+\frac{p q y^{2}}{p+2 q}-x\right)^{q}$.

Theorem 2. The quadratic-linear polynomial differential systems (3) satisfying (S2) and having a global analytic first integral $H$ are the following:

(a) $d=f=0$, ce $\neq 0$ and $b=0$ having $H=(c+e x)^{2 c} \exp \left(e^{2} y^{2}-2 e x\right)$;

(b) $e=f=0, c d \neq 0$ and $b=0$ having $H=\left(c+2 c d y+2 d^{2} x^{2}\right) \exp (-2 d y)$;

(c) $b=e=0$ and $f \neq 0$ having $H=\left(2 f(c d+f)+4 d^{3} f x^{2}+4 d f(c d+f) y+\right.$ $\left.4 d^{2} f^{2} y^{2}\right) \exp [-2 d(y+c /(2 f))]$. 
The proofs of Theorems 1 and 2 are given in Sections 3 and 4 respectively. In Section 2 we present some known results necessary for the proof of our theorems.

\section{Preliminary Results}

First we show that we can reduce the study of all quadratic-linear systems having a finite singular point to the two classes (S1) and (S2).

Proposition 3. Any quadratic-linear differential system

(4) $x^{\prime}=a_{00}+a_{10} x+a_{01} y+a_{20} x^{2}+a_{11} x y+a_{02} y^{2}, \quad y^{\prime}=A+B x+C y$, with $A^{2}+B^{2}+C^{2} \neq 0$ having a singular point, through a linear change of variables and a rescaling of the time can be written into the form

$$
x^{\prime}=b x+c y+d x^{2}+e x y+f y^{2}, \quad y^{\prime}=Q(x, y),
$$

where $Q(x, y)$ is either $x$ or $y$.

Proof. Assume that $B \neq 0$. Then doing the change of variables $X=A+B x+C y$, $Y=y$, system (4) becomes the system

$$
X^{\prime}=\bar{a}+\bar{b} X+\bar{c} Y+\bar{d} X^{2}+\bar{e} X Y+\bar{f} Y^{2}, \quad Y^{\prime}=Q(X, Y),
$$

with $Q(X, Y)=X$.

Suppose $B=0$ and $C \neq 0$. Then the change of variables $X=x, Y=A+C y$ and $T=C t$ writes system (4) into the form (6) with $Q(X, Y)=Y$.

Finally if $B=C=0$ then $A \neq 0$, and system (4) has no finite singular points.

Now let $(\alpha, \beta)$ be a singular point of system (6) with $Q(X, Y)$ equal to either $X$ or $Y$. When $Q(X, Y)=X$ the finite singular point is $(\alpha, \beta)$ with $\alpha=0$, and when $Q(X, Y)=Y$ it is $(\alpha, \beta)$ with $\beta=0$. So, doing the translation $x=X-\alpha$, $y=Y-\beta$ we obtain that system (6) becomes system (5), and the proposition is proved.

Now we shall introduce some auxiliary results that will be used through the paper. Let $X=X(x, y)=(P(x, y), Q(x, y))$ be the vector field associated to our system (3).

The following result is due to Poincaré in [1] and its proof can be found in [6].

Theorem 4. Assume that the eigenvalues $\lambda_{1} \neq 0$ and $\lambda_{2} \neq 0$ at some singular point of $X$ do not satisfy any resonance condition of the form

$$
\lambda_{1} k_{1}+\lambda_{2} k_{2}=0 \text { for } k_{1}, k_{2} \in \mathbb{Z}^{+} \text {with } k_{1}+k_{2}>0 .
$$

Then system (3) has no local analytic first integrals.

The following result is due to Li, Llibre and Zhang, see [12].

Theorem 5. Assume that the eigenvalues $\lambda_{1}$ and $\lambda_{2}$ at some singular point $(\bar{x}, \bar{y})$ of $X$ satisfy that $\lambda_{1}=0$ and $\lambda_{2} \neq 0$. Then system (3) has no local analytic first integrals if the singular point $(\bar{x}, \bar{y})$ is isolated.

We must mention that the singular points appearing in the statements of Theorems 4 and 5 can be real or complex, but our system (3) always is real.

On the other hand we need a result concerning with the characterization of the centers. This is due to Poincaré [16] and Liapunov [11], see also Moussu [15]. 
Theorem 6 (Nondegenerate Center Theorem). We consider the polynomial differential system on the plane of the form

$$
\dot{x}=-y+F(x, y) \quad \dot{y}=x+G(x, y),
$$

where $F$ and $G$ are polynomials formed by monomials of degree $\geq 2$. Then system (7) has a center at the origin if and only if there exists a local analytic first integral defined in a neighborhood of the origin.

We also need a result related with the classification theorem for centers of quadratic systems due to Kapteyn $[9,10]$ and Bautin [2]. We state it in complex notation.

Theorem 7. Any system (7) with $F$ and $G$ homogeneous polynomials of degree two candidate to have a center can be written in complex notation as

$$
z^{\prime}=i z+A z^{2}+B z \bar{z}+C \bar{z}^{2}, \quad A, B, C \in \mathbb{C},
$$

where $z=x+y i$. System (8) has a center at the origin if and only if one of the following four conditions hold.

(i) $B=0$,

(ii) $2 A+\bar{B}=0$,

(iii) $\operatorname{Im}(A B)=\operatorname{Im}\left(\bar{B}^{3} C\right)=\operatorname{Im}\left(A^{3} C\right)=0$,

(iv) $|C|-|B|=A-2 \bar{B}=0$, where $|\cdot|$ indicates the modulo of a complex number.

\section{Proof of Theorem 1}

Assume $d=0$. If $b=0$ then it is easy to check that the function $H$ given in Theorem 1(a) satisfies (2).

Assume $b \neq 0$ and $b \notin \mathbb{Q}^{-}$. Then $(0,0)$ is a singular point of system (S1) having eigenvalues $\lambda_{1}=b$ and $\lambda_{2}=1$. Since for all $k_{1}, k_{2} \in \mathbb{Z}^{+}$with $k_{1}+k_{2}>0$ we have $k_{1} b+k_{2} \neq 0$ (otherwise $b \in \mathbb{Q}^{-}$), by Theorem 4 the proposition follows.

Suppose now $b=-p / q$ with $p$ and $q$ positive integers, and $e \neq 0$ then an easy computation shows that

$$
H=e^{2+p / q} x y^{p / q} \exp (-e y)+c e \Gamma(1+p / q, e y)+f \Gamma(2+p / q, e y)
$$

is a first integral of system (S1) where

$$
\Gamma(u, z)=\int_{z}^{+\infty} t^{u-1} \exp (-t) d t
$$

is the plica function. Since the function $\Gamma(u, z)$ is not continuous when $z \in(-\infty, 0)$ we do not have a global analytic first integral.

Assume now $b=-p / q$ with $p$ and $q$ positive integers and $e=0$. Then the function $H$ given in Theorem 1(b) satisfies (2).

Now we assume that $d \neq 0$. In this case system (S1) has the singular points $(0,0)$ and $(-b / d, 0)$.

We first assume $b=0$. In this case only the origin is a singular point. Its eigenvalues are 0 and 1 . Since this singular point is isolated, Theorem 5 says that system (S1) has no analytic first integrals.

Now we assume $b \neq 0$ and $b \notin \mathbb{Q}^{+}$. In this case the eigenvalues of $(-b / d, 0)$ are $\lambda_{1}=-b$ and $\lambda_{2}=1$. So given $k_{1}, k_{2} \in \mathbb{Z}^{+}$with $k_{1}+k_{2}>0$, we have $-b k_{1}+k_{2} \neq 0$. Then Theorem 4 implies that system (S1) has no global analytic first integrals. 
Finally consider the case $b \in \mathbb{Q}^{+} \backslash\{0\}$. In this case the eigenvalues of $(0,0)$ are $\lambda_{1}=b$ and $\lambda_{2}=1$. So given $k_{1}, k_{2} \in \mathbb{Z}^{+}$with $k_{1}+k_{2}>0$, we have $b k_{1}+k_{2}>0$. Then Theorem 4 implies that system (S1) has no global analytic first integrals. This proves the theorem.

\section{ANALYTIC FIRST INTEGRALS FOR SYSTEM (S2)}

We shall prove Theorem 2 in the next propositions.

Proposition 8. Assume that $f=0, b c \neq 0$ and $c / b^{2} \notin \mathbb{Q}^{+}$. Then system (S2) has no local analytic first integrals in a neighborhood of zero.

Proof. We note that system (S2) has the singular point $(0,0)$. Its eigenvalues are

$$
\lambda_{1,2}=\frac{b \pm \sqrt{b^{2}+4 c}}{2} .
$$

Clearly

$$
\lambda_{1}+\lambda_{2}=b \quad \text { and } \quad \lambda_{1} \lambda_{2}=-c .
$$

Suppose that there exists positive integers $k_{1}, k_{2}$ such that $k_{1} \lambda_{1}+k_{2} \lambda_{2}=0$. Note that by Theorem 4 if such integers do not exist the proposition is proved. Then $\lambda_{1}=-\alpha \lambda_{2}$ with $\alpha$ a positive rational. The two equalities of (9) become

$$
b=(1-\alpha) \lambda_{2} \quad \text { and } \quad c=\alpha \lambda_{2}^{2} .
$$

Since we have

$$
-\frac{b^{2}}{c}=-\frac{(1-\alpha)^{2}}{\alpha} \in \mathbb{Q}^{-} .
$$

Note that $\alpha \neq 1$ because $b \neq 0$. Therefore, since $b^{2} / c \notin \mathbb{Q}^{+}$we cannot have $k_{1} \lambda_{1}+k_{2} \lambda_{2}=0$ and the proposition is proved.

Proposition 9. System (S2) with $f=0, b c \neq 0$ and $\alpha=c / b^{2} \in \mathbb{Q}^{+}$with $\alpha \neq$ $p q /(p-q)^{2}$ for some positive coprime integers $p, q$ with $p>q$ has no global analytic first integrals.

Proof. Doing the rescaling $(X, Y, T)=\left(\frac{c}{b^{2}} x, \frac{c}{b} y, b t\right)$ system (S2) becomes of the form

$$
x^{\prime}=x+\alpha y+\frac{d b}{c} x^{2}+\frac{e}{c} x y, \quad y^{\prime}=x,
$$

where we have written again $(x, y, t)$ instead of $(X, Y, T)$. We assume that $H=$ $H(x, y)$ is a local analytic first integral in a neighborhood of the origin. We write it as

$$
H=\sum_{k \geq 1} H_{k}(x, y)
$$

where each $H_{k}$ is a homogeneous polynomial of degree $k$. We will show by induction that

$$
H_{k}=0 \quad \text { for } k \geq 1 .
$$

Then clearly from (10) we will obtain that system (S2) has no global analytic first integrals, and the proof of the proposition is done.

Since $H$ is a first integral it must satisfy

$$
\left(x+\alpha y+\frac{d b}{c} x^{2}+\frac{e}{c} x y\right) \frac{\partial H}{\partial x}+x \frac{\partial H}{\partial y}=0 .
$$


Now we will do the induction. The terms of degree one in (12) must satisfy

$$
(x+\alpha y) \frac{\partial H_{1}}{\partial x}+x \frac{\partial H_{1}}{\partial y}=0 .
$$

Clearly, since $\alpha \neq 0$ we have that $\partial H_{1} / \partial x=0$. Then $\partial H_{1} / \partial y=0$ and thus $H_{1}=0$ which proves (19) for $k=1$. Now we assume that (19) is true for $k=1, \ldots, j-1$ with $j \geq 2$ and we will prove it for $k=j$. By the induction hypothesis, the terms of order $j$ in (12) must satisfy

$$
(x+\alpha y) \frac{\partial H_{j}}{\partial x}+x \frac{\partial H_{j}}{\partial y}=0
$$

Therefore, either $H_{j}=0$ or $H_{j}$ is a first integral of the linear system

$$
x^{\prime}=x+\alpha y, \quad y^{\prime}=x .
$$

Computing a first integral of this system we obtain that it must be a function of

$$
G=((\sqrt{1+4 \alpha}-1) x-2 \alpha y)^{1+\sqrt{1+4 \alpha}}(2 x+(\sqrt{1+4 \alpha}-1) y)^{-1+\sqrt{1+4 \alpha}}
$$

The unique possibility in order that $G^{n_{2}}$ be a polynomial is

$$
-1+\sqrt{1+4 \alpha}=\frac{n_{1}}{n_{2}} \quad \text { and } \quad 1+\sqrt{1+4 \alpha}=\frac{n_{3}}{n_{2}}, \quad n_{1}, n_{3} \in \mathbb{Z}^{+}, n_{3} \neq n_{1}
$$

Then we have that

$$
\frac{n_{1}}{n_{2}}+1=\frac{n_{3}}{n_{2}}-1, \quad \text { that is } \quad n_{2}=\frac{n_{3}-n_{1}}{2} .
$$

Note that $n_{2}$ is not necessarily an integer. Hence,

$$
\sqrt{1+4 \alpha}=\frac{n_{1}+n_{2}}{n_{2}}=\frac{n_{1}+n_{3}}{n_{3}-n_{1}},
$$

which yields

$$
\alpha=\frac{1}{4}\left(\left(\frac{n_{1}+n_{3}}{n_{3}-n_{1}}\right)^{2}-1\right)=\frac{n_{1} n_{3}}{\left(n_{3}-n_{1}\right)^{2}}, \quad n_{1}, n_{3} \in \mathbb{Z}^{+}, n_{1} \neq n_{3},
$$

a contradiction, and hence the induction process has ended. Note that $n_{3}>n_{1}$. Furthermore, $n_{1}$ and $n_{3}$ are coprime since otherwise, setting $n_{1}=\operatorname{g.c} . \mathrm{d}\left\{n_{1}, n_{3}\right\} \hat{n}_{1}$ and $n_{3}=$ g.c.d $\left\{n_{1}, n_{3}\right\} \hat{n}_{3}$ we get

$$
\alpha=\frac{\text { g.c.d }\left\{n_{1}, n_{3}\right\}^{2} \hat{n}_{1} \hat{n}_{3}}{\left(\text { g.c.d }\left\{n_{1}, n_{3}\right\} \hat{n}_{1}+\text { g.c. } d\left\{n_{1}, n_{3}\right\} \hat{n}_{3}\right)^{2}}=\frac{\hat{n}_{1} \hat{n}_{3}}{\left(\hat{n}_{1}-\hat{n}_{3}\right)^{2}} .
$$

Proposition 10. System (S2) with $f=0, b c \neq 0, \alpha=c / b^{2}$ with $\alpha=p q /(p-q)^{2}$ for some positive coprime integers $p, q$ with $p>q$ and $d \neq-e(p-q) /(b p)$ has no global analytic first integrals.

Proof. We consider system (S2) with $f=0, c=b^{2} p q /(p-q)^{2}$ where $p, q$ are coprime positive integers with $p>q$ and $d \neq-e(p-q) /(b p)$. Under these assumptions and doing the rescaling $(X, Y, T)=\left(\frac{c}{b^{2}} x, \frac{c}{b} y, b t\right)$ we have

(13) $x^{\prime}=x+\frac{p q}{(p-q)^{2}} y+D x^{2}+E x y, \quad y^{\prime}=x, \quad D=\frac{d(p-q)^{2}}{b p q}, \quad E=\frac{e(p-q)^{2}}{b^{2} p q}$ 
with $p D+E(p-q) \neq 0$ and where we have written again $(x, y, t)$ instead of $(X, Y, T)$. Making the change of variables

(14) $u=(p-q) x+q y, \quad v=(q-p) x+p y, \quad$ i.e. $\quad x=\frac{p u-q v}{(p-q)(p+q)}, \quad y=\frac{u+v}{q-p}$

we have that system (13) becomes

$$
\begin{aligned}
u^{\prime} & =\frac{p}{p-q} u+\frac{p(D p+E(p-q)) u^{2}}{(p-q)(p+q)^{2}}+\frac{\left(E(p-q)^{2}-2 D p q\right) u v}{(p-q)(p+q)^{2}}+ \\
& \frac{q(D q+E(q-p)) v^{2}}{(p-q)(p+q)^{2}}, \\
v^{\prime} & =-\frac{q v}{p-q}-\frac{p(D p+E(p-q)) u^{2}}{(p-q)(p+q)^{2}}+\frac{\left(2 D p q-E(p-q)^{2}\right) u v}{(p-q)(p+q)^{2}}+ \\
& \frac{q(E(p-q)-D q) v^{2}}{(p-q)(p+q)^{2}} .
\end{aligned}
$$

We change from the variables $(u, v)$ to the variables $(u, T)$ where

$$
T=u^{q} v^{p}, \quad \text { that is } v=T^{1 / p} u^{-q / p} .
$$

Then we have from (15) that

$$
\begin{aligned}
u^{\prime} & =\frac{p u}{p-q}+\frac{p(D p+E(p-q)) u^{2}}{(p-q)(p+q)^{2}}+\frac{q(D q+E(q-p)) u^{-\frac{2 q}{p}} T^{2 / p}}{(p-q)(p+q)^{2}}+ \\
& \frac{\left(E(p-q)^{2}-2 D p q\right) u^{\frac{p-q}{p}} T^{\frac{1}{p}}}{(p-q)(p+q)^{2}}, \\
T^{\prime} & =\frac{u^{\frac{q-p}{p}} T^{\frac{p-1}{p}}}{(p-q)(p+q)^{2}}\left(p u-q u^{-\frac{q}{p}} T^{\frac{1}{p}}\right)^{2}\left((E(p-q)-D q) u^{-\frac{q}{p}} T^{\frac{1}{p}}+(D p+E(p-q)) u\right) .
\end{aligned}
$$

Let $H=H(x, y)$ be a formal first integral of system (13). Then $\hat{H}(u, v)=H(x, y)$ is a formal first integral of system (15) and $\tilde{H}(u, T)=\hat{H}(u, v)$ is a formal first integral of system (17). Writing $\hat{H}(u, v)=\sum_{j \geq 0} H_{j}(u) v^{j}$ with $H_{j}$ a formal series in $u$, we can write $\tilde{H}(u, T)$ as

$$
\tilde{H}=\tilde{H}(u, T)=\sum_{j \geq 0} \tilde{H}_{j}(u) T^{j / p},
$$

where $\tilde{H}_{j}(u)=H_{j}(u) u^{-j q / p}$. Since $\tilde{H}$ is a first integral we can assume that it has no constant term. Note that $\tilde{H}$ satisfies

$$
T^{\prime} \frac{\partial \tilde{H}}{\partial T}+u^{\prime} \frac{\partial \tilde{H}}{\partial u}=0,
$$

with $\left(T^{\prime}, v^{\prime}\right)$ as in (17). We will show by induction that

$$
\tilde{H}_{j}(u)=0 \text { for } j \geq 0 .
$$

Note that to conclude the proof of the proposition it is enough to show that (19) holds.

First we note that equation (18) restricted to $T=0$ becomes

$$
\left(\frac{p u}{p-q}+\frac{p(D p+E(p-q)) u^{2}}{(p-q)(p+q)^{2}}\right) \tilde{H}_{0}^{\prime}(u)=0,
$$


where the prime indicates derivative with respect to the variable $u$. Thus $\tilde{H}_{0}$ is a constant. Since $\tilde{H}$ has no constant terms we get $\tilde{H}_{0}=0$. This proves (19) for $j=0$.

We assume that (19) is satisfied for $j=0, \ldots, n-1$ with $n \geq 1$ and we shall prove it for $j=n$. By the induction hypothesis we have

$$
\tilde{H}=\sum_{j \geq 0} \tilde{H}_{j+n}(u) T^{(j+n) / p}=T^{n / p} g(u, T),
$$

with $g(u, 0)=\tilde{H}_{n}(u)$. Now after simplifying equation (18) by $T^{(n-1) / p}$, and after restricting it to $T=0$, equation (18) becomes

$$
\frac{n u^{(q+p) / p}}{(p-q)(p+q)^{2}}(D p+E(p-q)) \tilde{H}_{n}(u)=0 .
$$

Therefore $\tilde{H}_{n}(u)=0$. This proves equation (19) for $j=n$. In short, the proposition is proved.

Proposition 11. System (S2) with $f=0, b c \neq 0, \alpha=c / b^{2}$ with $\alpha=p q /(p-q)^{2}$ for some positive coprime integers $p, q$ with $p>q$ and $d=-e(p-q) /(b p)$ has no global analytic first integrals.

Proof. We consider system (S2) with $f=0, b=\sqrt{c}(p-q) / \sqrt{p q}$ where $p, q$ are coprime positive integers with $p>q$ and $d=-e(p-q) /(b p)$. Under these assumptions and doing the rescaling $(X, Y, T)=\left(\frac{c}{b^{2}} x, \frac{c}{b} y, b t\right)$ we have

$$
x^{\prime}=x+\frac{p q}{(p-q)^{2}} y-\frac{p-q}{p} E x^{2}+E x y, \quad y^{\prime}=x, \quad E=\frac{e(p-q)^{2}}{b^{2} p q},
$$

where we have written again $(x, y, t)$ instead of $(X, Y, T)$. Making the change of variables as in (14) we have that system (20) becomes

$$
\begin{aligned}
& u^{\prime}=\frac{p u}{p-q}-\frac{E(p-1) p u^{2}}{(p+q)^{2}}+\frac{E(2 q p+p-q) v u}{(p+q)^{2}}-\frac{E q(q+1) v^{2}}{(p+q)^{2}}, \\
& v^{\prime}=-\frac{q v}{p-q}+\frac{E(p-1) p u^{2}}{(p+q)^{2}}+\frac{E(q-p(2 q+1)) v u}{(p+q)^{2}}+\frac{E q(q+1) v^{2}}{(p+q)^{2}} .
\end{aligned}
$$

We change from the variables $(u, v)$ to the variables $(u, T)$ as in $(16)$. Then we have from (21) that

$$
\begin{aligned}
& u^{\prime}=\frac{p u}{p-q}-\frac{E(p-1) p u^{2}}{(p+q)^{2}}-\frac{E q(q+1) u^{-\frac{2 q}{p}} T^{2 / p}}{(p+q)^{2}}+\frac{E(2 q p+p-q) u^{\frac{p-q}{p}} T^{\frac{1}{p}}}{(p+q)^{2}} \\
& T^{\prime}=\frac{E}{(p+q)^{2}} u^{\frac{q-p}{p}} T^{\frac{p-1}{p}}\left(p u-q u^{-\frac{q}{p}} T^{\frac{1}{p}}\right)^{2}\left((p-1) u-u^{-\frac{q}{p}} T^{\frac{1}{p}}-q u^{-\frac{q}{p}} T^{\frac{1}{p}}\right)
\end{aligned}
$$

Let $H=H(x, y)$ be a formal first integral of system (13). Then proceeding as in the proof of Proposition 10 we have $\tilde{H}(u, T)=H(x, y)$ and we write it as

$$
\tilde{H}=\tilde{H}(u, T)=\sum_{j \geq 0} \tilde{H}_{j}(u) T^{j / p},
$$

where $\tilde{H}_{j}(u)=H_{j}(u) u^{-j q / p}$. Since $\tilde{H}$ is a first integral we can assume that it has no constant term. Note that $\tilde{H}$ satisfies (18). We will show by induction that

$$
\tilde{H}_{j}(u)=0 \text { for } j \geq 0
$$


Note that to conclude the proof of the proposition it is enough to show that (23) holds.

First we note that equation (18) restricted to $T=0$ becomes

$$
\left(\frac{p u}{p-q}-\frac{E(p-1) p u^{2}}{(p+q)^{2}}\right) \tilde{H}_{0}^{\prime}(u)=0
$$

where the prime indicates derivative with respect to the variable $u$. Thus, $\tilde{H}_{0}$ is a constant. Since $\tilde{H}$ has no constant terms we get $\tilde{H}_{0}=0$. This proves (23) for $j=0$.

We assume that (23) is satisfied for $j=0, \ldots, n-1$ with $n \geq 1$ and we shall prove it for $j=n$. By the induction hypothesis we have

$$
\tilde{H}=\sum_{j \geq 0} \tilde{H}_{j+n}(u) T^{(j+n) / p}=T^{n / p} g(u, T),
$$

with $g(u, 0)=\tilde{H}_{n}(u)$. Now after simplifying equation (18) by $T^{(n-1) / p}$, and after restricting it to $T=0$, equation (18) becomes

$$
-\frac{n E u^{(q+p) / p}}{(p-q)(p-q)^{2}}(p-1) \tilde{H}_{n}(u)=0 .
$$

Since $p>q \geq 1$ and $? \neq 0$ (otherwise system (3) would be linear) we have that $\tilde{H}_{n}(u)=0$. This proves equation $(23)$ for $j=n$. In short, the proposition is proved.

Proposition 12. Assume that in system (S2) we have $f=0, c \neq 0$ and $b=0$.

(a) If $d=0$ and $e \neq 0$, then the function $H$ of Theorem 2(a) satisfies (2).

(b) If $e=0$ and $d \neq 0$, then the function $H$ of Theorem 2(b) satisfies (2).

(c) If de $\neq 0$, then it has no global analytic first integrals.

Proof. From (S2) we get that

$$
x^{\prime}=c y+d x^{2}+e x y, \quad y^{\prime}=x .
$$

Statements (a) and (b) follows easily by direct computations.

Assume $d e \neq 0$ and $c<0$. Then system (S2) becomes

$$
x^{\prime}=-|c| y+d x^{2}+e x y, \quad y^{\prime}=x .
$$

Doing the change of variables

$$
Z=\frac{1}{\sqrt{|c|}} x, \quad W=y \text { and } t=\frac{1}{\sqrt{|c|}} \tau
$$

we obtain

$$
Z^{\prime}=-W+d Z^{2}+\frac{e}{\sqrt{|c|}} Z W, \quad W^{\prime}=Z
$$

where now the prime indicates derivative with respect to $\tau$. We note that system (25) can be written as in (8) taking

$$
z=Z+W i, \quad A=\frac{d}{4}-\frac{e}{4 \sqrt{|c|}} i, \quad B=\frac{d}{2}, \quad C=\frac{d}{4}+\frac{e}{4 \sqrt{|c|}} i .
$$

It is clear that $B \neq 0,2 A+\bar{B} \neq 0, A-2 \bar{B} \neq 0$ and $\operatorname{Im}(A B) \neq 0$. Then by Theorem 7 we have that system (25) has not a center at the origin, and by Theorem 6 then system (25) has no local analytic first integrals in a neighborhood of the origin. 
Consequently system (S2) with $d e \neq 0$ and $c<0$ has no global analytic first integrals.

Now suppose $d e \neq 0$ with $c>0$, then system (S2) becomes

$$
x^{\prime}=|c| y+d x^{2}+e x y, \quad y^{\prime}=x .
$$

Using the change of variables (24) this system goes over to

$$
Z^{\prime}=W+d Z^{2}+\frac{e}{\sqrt{|c|}} Z W, \quad W^{\prime}=Z,
$$

System (26) can be written as in (8) taking

$$
z=Z+W, \quad \bar{z}=Z-W, \quad t=i \tau, \quad A=\frac{d}{4}+\frac{e}{4 \sqrt{|c|}} i, \quad B=\frac{d}{2}, \quad C=\frac{d}{4}-\frac{e}{4 \sqrt{|c|}} i .
$$

The same arguments used in the case $c<0$ works now for proving that system (26) has no local analytic first integrals in a neighborhood of the origin (see [14] for details). Consequently system (S2) with $d e \neq 0$ and $c>0$ has no global analytic first integrals. This completes the proof of statement (c).

Now we are left with system (S2) with $f \neq 0$. Doing the change of variables

$$
x=X, \quad y=Y-\frac{c}{2 f}
$$

we transform system (S2) with $f \neq 0$ into the following system

(S2") $X^{\prime}=\tilde{a}+\tilde{b} X+d X^{2}+e X Y+f Y^{2}, Y^{\prime}=X$, with $\tilde{a}=-\frac{c^{2}}{4 f}$ and $\tilde{b}=b-\frac{c e}{2 f}$.

Now we will work with system (S2") instead of system (S2).

Proposition 13. If $f \neq 0$ and $b=e=0$ then

$$
H=\exp (-2 d Y)\left(-c^{2} d^{2}+2 f^{2}+4 d^{3} f X^{2}+4 d f^{2} Y+4 d^{2} f^{2} Y^{2}\right)
$$

is a global analytic first integral of system (S2"), and we obtain the global analytic first integral of Theorem 2(c).

Proof. The proof follows with a direct calculation.

Proposition 14. System (S2') with $f \neq 0, \tilde{b}^{2}+e^{2} \neq 0$ and $c \neq 0$ has no global analytic first integrals.

Proof. In this case the singular points of (S2') are $u_{ \pm}=(0, \pm \sqrt{-\tilde{a} / f})$ because $\tilde{a} f<0$. We separate the proof in two cases.

Case 1: $\tilde{b}-e \sqrt{-\tilde{a} / f} \neq 0$. Since the eigenvalues at $u_{-}$are

$$
\lambda_{1,2}=\frac{\tilde{b}-e \sqrt{-\tilde{a} / f} \pm \sqrt{(\tilde{b}-e \sqrt{-\tilde{a} / f})^{2}-8 \sqrt{-\tilde{a} f}}}{2},
$$

both eigenvalues have either positive or negative real parts. So given $k_{1}, k_{2} \in \mathbb{Z}^{+}$ with $k_{1}+k_{2}>0$, we have $\lambda_{1} k_{1}+\lambda_{2} k_{2} \neq 0$. Then Theorem 4 implies that system (S2") has no global analytic first integrals.

Case 2: $\tilde{b}-e \sqrt{-\tilde{a} / f}=0$. We take $\tilde{b}=e \sqrt{-\tilde{a} / f}$. We translate the singular point $u_{-}$at the origin, we continue denoting the new variables as $(X, Y)$, and doing the change of variables

$$
x=\frac{X}{\sqrt{2 \sqrt{-\tilde{a} f}}}, \quad y=Y, \quad T=\sqrt{2 \sqrt{-\tilde{a} f}} t
$$


system (S2") becomes

$$
\dot{x}=-y+d x^{2}-\frac{e(-\tilde{a} f)^{3 / 4}}{\sqrt{2} \tilde{a} f} x y-\frac{\sqrt{-\tilde{a} f}}{2 \tilde{a}} y^{2}, \quad \dot{y}=x .
$$

We note that this system can be written as in (8) taking

$$
z=x+y i, \quad A=\frac{2 \tilde{a} d+\sqrt{-\tilde{a} f}}{8 \tilde{a}}+\frac{e(-\tilde{a} f)^{3 / 4}}{4 \sqrt{2} \tilde{a} f} i, \quad B=\frac{2 \tilde{a} d-\sqrt{-\tilde{a} f}}{4 \tilde{a}}, \quad C=\bar{A} .
$$

Here $\bar{A}$ denotes the conjugate of $A$. It is clear that $2 A+\bar{B} \neq 0, A-2 \bar{B} \neq 0$ and $\operatorname{Im}(A B) \neq 0$. Then by Theorem 7 we have that system (27) has not a center at the origin, and by Theorem 6 then system (27) has no local analytic first integrals in a neighborhood of the origin. Consequently system (S2") in this case has no global analytic first integrals.

Proposition 15. System (S2") with $f \neq 0, \tilde{b}^{2}+e^{2} \neq 0$ and $c=0$ has no global analytic first integrals.

Proof. Since $\tilde{a}=0$ system (S2") has a unique singular point, the origin. Its eigenvalues are $\lambda=0$ and $\lambda=\tilde{b}$. If $\tilde{b} \neq 0$ then the origin is isolated, consequently Theorem 5 says that system (S2") has no analytic first integrals.

Now we consider the case $\tilde{b}=0$. Then by hypothesis $e \neq 0$ and furthermore system (S2") becomes

$$
X^{\prime}=d X^{2}+e X Y+f Y^{2}, \quad Y^{\prime}=X .
$$

We claim that system (28) has no global analytic first integrals. We note that the proof of the proposition follows from the claim. Now we shall prove the claim.

Let $H=H(X, Y)=\sum_{k \geq 1} H_{k}(X, Y)$ be a first integral of (28), where $H_{k}$ is a homogeneous polynomial of degree $k$. Then $H$ satisfies

$$
\left(d X^{2}+e X Y+f Y^{2}\right) \frac{\partial H}{\partial X}+X \frac{\partial H}{\partial Y}=0 .
$$

Computing the terms with different degree in (29) we get

$$
X \frac{\partial H_{k}}{\partial Y}=-\left(d X^{2}+e X Y+f Y^{2}\right) \frac{\partial H_{k-1}}{\partial X}, \quad \text { for } k \geq 1 .
$$

We will show by induction that for $k \geq 1$,

$$
H_{k-1}=0 \quad \text { and } \quad \frac{\partial H_{k}}{\partial Y}=0
$$

We note that (31) clearly implies that $H=0$ a contradiction with the fact that $H$ is a global first integral of system (28). Hence the claim will be proved if we prove the induction hypothesis.

For $k=1(30)$ yields $\partial H_{1} / \partial Y=0$, and since $H_{0}=0$ the induction hypothesis is proved for $k=1$.

Now we assume that (31) is true for $k=1, \ldots, l(l \geq 1)$ and we will prove it for $k=l+1$. By the induction hypothesis we have

$$
H_{l-1}=0 \quad \text { and } \quad H_{l}=a_{l} X^{l}, \quad a_{l} \in \mathbb{R} .
$$

Equation (30) with $k=l+1$ yields

$$
X \frac{\partial H_{l+1}}{\partial Y}=-a_{l} l\left(d X^{2}+e X Y+f Y^{2}\right) X^{l-1},
$$


If $l=1$ then (32) becomes

$$
X \frac{\partial H_{2}}{\partial Y}=-a_{1}\left(d X^{2}+e X Y+f Y^{2}\right)
$$

From this equation since $f \neq 0$ we get that $a_{1}=0$ and $\partial H_{2} / \partial Y$, so $H_{1}=0$ and $H_{2}=a_{2} X^{2}$. The induction hypothesis is proved for $k=2$. Now assume $l \geq 2$.

First we will prove by induction that for $m \geq 1$,

(33) $H_{l+m}=(-1)^{m} f^{m-1}\left(a_{l} f C_{l, m} Y+a_{l} e K_{l, m} X\right) Y^{3 m-1} X^{l-2 m}+O\left(X^{l-2 m+2}\right)$, where $C_{l, m}$ and $K_{l, m}$ are positive constants depending on $l$ and $m$. This result will allow us to complete the proof of the induction hypothesis (31).

Since $l \geq 2$ solving $(32)$ we get

$$
\begin{aligned}
H_{l+1} & =-a_{l} l X^{l-2}\left(d X^{2} Y+\frac{e}{2} X Y^{2}+\frac{f}{3} Y^{3}\right)+a_{l+1} X^{l+1} \\
& =-a_{l} f C_{l, 1} X^{l-2} Y^{3}-a_{l} e K_{l, 1} X^{l-1} Y^{2}+O\left(X^{l}\right),
\end{aligned}
$$

where $C_{l, 1}=l / 3$ and $K_{l, 1}=l / 2$. This proves (33) with $m=1$. Now we assume that (33) is true for $m=1, \ldots, n-1(n \geq 2)$ and we will prove it for $m=n$. By the induction hypothesis and (30) with $k=l+n$ we have

$$
\begin{aligned}
X \frac{\partial H_{l+n}}{\partial Y}= & -\left(d X^{2}+e X Y+f Y^{2}\right) \frac{\partial H_{l+n-1}}{\partial X} \\
= & -\left(d X^{2}+e X Y+f Y^{2}\right)\left[(-1)^{n-1} a_{l} f^{n-1} C_{l, n-1}(l-2 n+2) X^{l-2 n+1} Y^{3 n-3}\right. \\
& \left.+(-1)^{n-1} a_{l} e f^{n-2} K_{l, n-1}(l-2 n+3) X^{l-2 n+2} Y^{3 n-4}+O\left(X^{l-2 n+3}\right)\right] \\
= & (-1)^{n} a_{l} f^{n} C_{l, n-1}(l-2 n+2) X^{l-2 n+1} Y^{3 n-1} \\
& +(-1)^{n} a_{l} e f^{n-1} K_{l, n-1}(l-2 n+3) X^{l-2 n+2} Y^{3 n-2} \\
& +(-1)^{n} a_{l} e f^{n-1} C_{l, n-1}(l-2 n+2) X^{l-2 n+2} Y^{3 n-2}+O\left(X^{l-2 n+3}\right) \\
= & (-1)^{n} a_{l} f^{n} C_{l, n-1}(l-2 n+2) X^{l-2 n+1} Y^{3 n-1} \\
& +(-1)^{n} a_{l} e f^{n-1}\left[K_{l, n-1}(l-2 n+3)+C_{l, n-1}(l-2 n+2)\right] X^{l-2 n+2} Y^{3 n-2} \\
& +O\left(X^{l-2 n+3}\right) .
\end{aligned}
$$

Therefore

$$
\begin{aligned}
\frac{\partial H_{l+n}}{\partial Y}= & (-1)^{n} a_{l} f^{n} C_{l, n-1}(l-2 n+2) X^{l-2 n} Y^{3 n-1} \\
& +(-1)^{n} a_{l} e f^{n-1}\left[K_{l, n-1}(l-2 n+3)+C_{l, n-1}(l-2 n+2)\right] X^{l-2 n+1} Y^{3 n-2} \\
& +O\left(X^{l-2 n+2}\right)
\end{aligned}
$$

which yields

$H_{l+n}=(-1)^{n} a_{l} f^{n} C_{l, n} X^{l-2 n} Y^{3 n}+(-1)^{n} a_{l} e f^{n-1} K_{l, n} X^{l-2 n+1} Y^{3 n-1}+O\left(X^{l-2 n+2}\right)$, with

$C_{l, n}=\frac{l-2 n+2}{3 n} C_{l, n-1}>0, \quad K_{l, n}=\frac{K_{l, n-1}(l-2 n+3)+C_{l, n-1}(l-2 n+2)}{3 n-1}>0$.

Therefore (33) is proved.

Now we continue with the proof of the induction hypothesis (31). In fact we shall prove that $a_{l}=0$ for $l \geq 2$. Then $H_{l}=0$ and from (29) we have that $\partial H_{l+1} / \partial Y=0$. This proves (31). We distinguish two cases. 
We first assume $l$ is odd. Then by (33) with $m=(l-1) / 2$ we obtain that

$$
H_{(3 l-1) / 2}=(-1)^{(l-1) / 2} a_{l} f^{(l-1) / 2} C_{l,(l-1) / 2} X Y^{(3 l-3) / 2}+O\left(X^{2}\right) .
$$

Then by $(30)$ with $k=(3 l+1) / 2$ we get

$$
\begin{aligned}
& X \frac{\partial H_{(3 l+1) / 2}}{\partial Y} \\
& =-\left(d X^{2}+e X Y+f Y^{2}\right)\left((-1)^{(l-1) / 2} a_{l} f^{(l-1) / 2} C_{l,(l-1) / 2} Y^{(3 l-3) / 2}+O(X)\right) .
\end{aligned}
$$

Now setting $X=0$ in (35) we get that $a_{l}=0$.

Finally, if $l$ is even, then (33) with $m=l / 2$ yields

$$
\begin{aligned}
H_{3 l / 2}= & (-1)^{l / 2} a_{l} f^{l / 2} C_{l, l / 2} Y^{3 l / 2} \\
& +(-1)^{l / 2} a_{l} e f^{(l-2) / 2} K_{l, l / 2} X Y^{(3 l-2) / 2}+O\left(X^{2}\right) .
\end{aligned}
$$

Then by $(30)$ with $k=(3 l+2) / 2$ we get

$X \frac{\partial H_{(3 l+2) / 2}}{\partial Y}=-\left(d X^{2}+e X Y+f Y^{2}\right)\left((-1)^{l / 2} a_{l} e f^{(l-2) / 2} K_{l, l / 2} Y^{(3 l-2) / 2}+O(X)\right)$.

Now taking $X=0$ in the previous expression we get that $a_{l}=0$.

\section{ACKNOWLEDGMENTS}

The first author has been supported by the grants MEC/FEDER MTM 200803437 and CIRIT 2005SGR 00550. The second author has been partially supported by the FCT through CAMGSD, Lisbon.

\section{REFERENCES}

1. D.V. Anosov And V.I. Arnold, Dynamical Systems I, Springer-Verlag, Berlin, 1988.

2. N. N. BAUtin, On the number of limit cycles which appear with the variation of the coefficients from an equilibrium position of focus or center type, Math. USSR-Sb. 100 (1954), 397-413.

3. L. CAiró, J. Llibre, Phase portraits of planar semi-homogeneous systems I, Nonlinear Analysis, Theory, Methods and Applications, 29, (1997), 783-811.

4. J. Chavarriga, B. Garcia, J. Llibre, J. S. Perez del Rio and J. A. Rodriguez, Polynomial first integrals of quadratic vector fields, J. Differential Equations 230 (2006), 393-421.

5. J. Chavarriga, H. Giacomini, J. Giné and J. Llibre, On the integrability of two-dimensional flows, J. Differential Equations 157 (1999), 163-182.

6. S.D. FuRTA, On non-integrability of general systems of differential equations, Z. Angew. Math. Phys. 47 (1996), 112-131.

7. A. Gasull, Sheng Li-Ren and J. Llibre, Chordal quadratic systems, Rocky Mountain J. of Math. 16 (1986), 751-782.

8. E. A. GonzÁlez, Generic properties of polynomial vector fields at infinity, Trans. Amer. Math. Soc. 143 (1969), 201-222.

9. W. KAPteyn, On the midpoints of inetgral curves of differential equations of the first degree, Nederl. Akad. Wetensch. Verslag. Afd. Natuurk. Konikl. Nederland (1911), 1446-1457 (Dutch).

10. W. KAPtEYN, New investigations on the midpoints of integrals of differential equations of the first degree, Nederl. Akad. Wetensch. Verslag. Afd. Natuurk. Konikl. Nederland 20 (1912), 1354-1365, 21 27-33 (Dutch).

11. A.M. Liapunov, Problème général de la stabilité du mouvement, Ann. of Math. Studies 17, Princeton Univ. Press, 1949.

12. W. Li, J. LliBre AND X. ZHANG, Local first integrals of differential systems and diffeomorphisms, Z. Angew. Math. Phys. 54 (2003), 1-21.

13. J. LlibRe AND C. VAlls, Global analytic first integrals for the real planar Lotka-Volterra systems, J. Math. Phys. 48, (2007), 1-13.

14. P. Joyal and C. Rousseau, Saddle quantities and applications, J. Diff. Equations 78 (1989), 374-399. 
15. R. Moussu, Symétrie et forme normale des centres et foyers dégénérés, Ergodic Theory and Dynamical systems 2 (1982), 241-251.

16. H. Poincaré, Mémoire sur les courbes définies par les équations différentielles, Oeuvreus de Henri Poincaré, Vol. I, Gauthiers-Villars, Paris, 1951, pp. 95-114.

17. J. W. REYN, A bibliography of the qualitative theory of quadratic systems of differential equations in the plane, Delf University of Technology, http://ta.twi.tudelft.nl/DV/Staff/ J.W.Reyn.html, 1997.

18. Ye YAnQIAn And others, Theory of Limit Cycles,Transl. Math. Monographs 66, Amer. Math. Soc., Providence, 1984.

19. Ye Yanqian, Qualitative Theory of Polynomial Differential Systems, Shanghai Scientific \& Technical Publishers, Shanghai, 1995 (in Chinese).

1 Departament de Matemàtiques, Universitat Autònoma de Barcelona, 08193 Bellaterra, Barcelona, Catalonia, Spain

E-mail address: jllibre@mat.uab.cat

2 Departamento de Matemática, Instituto Superior Técnico, Av. Rovisco Pais 1049001, Lisboa, Portugal

E-mail address: cvalls@math.ist.utl.pt 